\title{
Preparação e Caracterização de Materiais Híbridos de Óxidos de Ferro/Polianilina por Polimerização in situ
}

\author{
Taciano P. Ferreira \& Olacir A. Araújo
}

A polianilina é um polímero condutor que apresenta propriedades elétricas interessantes, pois possui um mecanismo especial de reação chamada de dopagem, que é um processo de transição de um polímero isolante ou semi-condutor para um material condutor. As ferritas são materiais cerâmicos com propriedades magnéticas associadas a sua estrutura cúbica do tipo espinélio, que apresenta formula geral $\mathrm{AB}_{2} \mathrm{O}_{4}$. Estes materiais têm sido largamente estudados devido às suas características de absorverem a radiação eletromagnética produzidas pelas inovações tecnológicas. Os materiais híbridos de polímeros com cargas inorgânicas podem apresentar novas propriedades devido a diferentes contribuições de cada um dos compostos em sua propriedade resultante. Neste trabalho foram desenvolvidos rotas de síntese para produção de híbridos de óxidos de ferro e polianilina (PAni), sendo que a PAni foi dopada com o ácido dodecilbenzenosulfônico (ADBS). Estes materiais foram caracterizados por difração de raios X, espectroscopia de IV, espectroscopia Mössbauer, microscopia eletrônica de transmissão (MET) e análise termogravimétrica.

Palavras-chave: polianilina; óxido de ferro; híbrido.

Polyaniline is a conducting polymer that presents interesting electrical properties, because it has a special mechanism called the doping reaction, a transition process from an insulating polymer or semi-conductor to a conductive material. Ferrites are ceramic materials with magnetic properties associated with its cubic structure of spinel type, with $\mathrm{AB}_{2} \mathrm{O}_{4}$ as the general formula. These materials have been widely studied due to their characteristic of absorbing the electromagnetic radiation produced by technological innovations. The hybrid polymeric materials with inorganic fillers may show new properties due to different contributions of each compounds. We have developed synthetic routes to produce hybrids of iron oxides and polyaniline (PAni), doped with sodium dodecylbenzenesulfonate (DBSA). These materials were characterized by X-ray diffraction, IR spectroscopy, Mössbauer spectroscopy, transmission electron microscopy (TEM) and thermogravimetric analysis.

Keywords: polyaniline; iron oxides; hybrid. 


\section{Introdução}

As ferritas são materiais cerâmicos com propriedades magnéticas, sendo as estruturas do tipo espinélio inverso as mais estudadas tecnologicamente, devido as suas propriedades relevantes como: altos valores de resistividade e permeabilidade magnética inicial, baixos valores de perdas por histerese e boa absorção de radiação eletromagnética. Elas são normalmente empregadas em núcleos de transformadores e indutores de equipamentos eletrônicos que utilizam dispositivos de alta freqüência ${ }^{4,13,20,24 .}$

Os polímeros convencionais apresentam, em sua maioria, alta resistividade elétrica, sendo uma característica necessária para diversas aplicações nos setores elétrico e eletrônico. Os materiais com estas propriedades apresentam o inconveniente de acumular energia estática, proveniente do fluxo de elétrons quando duas superfícies são colocadas em contato, sendo que, quando estes corpos são separados, um ficará carregado positivamente e o outro negativamente. Ao contrário dos materiais metálicos que dissipam esta energia, os materiais isolantes acumulam estas cargas na forma de energia estática. Na década de 70 iniciou-se o estudo de uma nova categoria de polímeros, os polímeros intrinsecamente condutores, que apresentam propriedades elétricas com potencial aplicação tecnológica ${ }^{1,14,17,23}$.

Atualmente os Materiais Absorvedores de Radiação Eletromagnética (MARE) têm sido largamente estudados devido a sua característica de absorver a radiação eletromagnética incidente, convertendo-a em energia térmica e com isto eliminando ou atenuando os níveis de radiações eletromagnéticas produzidas ${ }^{6}$. Os materiais híbridos de polímeros condutores com nanopartículas magnéticas têm chamado a atenção dos pesquisadores por apresentar propriedades magnéticas e elétricas. Estes materiais apresentam boa estabilidade ambiental, alta condutividade elétrica, baixa densidade e custos reduzidos ${ }^{16,18,19,26}$. Neste trabalho foram preparados e caracterizados novos materiais híbridos de magnetita óxidos de ferro com polianilina.

\section{Materiais e Métodos}

O método consistiu em preparar uma solução de concentração 0,15 mol. $L^{-1}$ de íons ferro, com proporção de 1:2 em mols de $\mathrm{Fe}^{2+}: \mathrm{Fe}^{3+}$, na forma de sulfato ferroso $\left(\mathrm{FeSO}_{4} \cdot 7 \mathrm{H}_{2} \mathrm{O}\right)$ e sulfato férrico $\left(\mathrm{Fe}_{2}\left(\mathrm{SO}_{4}\right)_{3} \cdot 16 \mathrm{H}_{2} \mathrm{O}\right)$, respectivamente. Preparou-se outra solução de hidróxido de amônio $\left(\mathrm{NH}_{4} \mathrm{OH}\right)$ com concentração de 1,5 mol.L-1. A síntese das partículas foi promovida pelo método de coprecipitação em meio aquoso básico, através de reação de condensação ${ }^{7}$. Preparou-se $1 \mathrm{~L}$ das soluções de íons ferro, posteriormente transferiu-se para um balão de fundo redondo de 3 bocas com capacidade de $2 \mathrm{~L}$, sendo submetido a agitação mecânica. O sistema foi estabilizado em $200 \mathrm{rpm}$, e resfriado com o auxílio de banho de gelo com sal grosso, à temperatura de $4-6^{\circ} \mathrm{C}$. Posteriormente $266,7 \mathrm{~mL}$ da solução de hidróxido de amônio foram adicionados gota a gota por aproximadamente $30 \mathrm{~min}$. A agitação permaneceu por $1 \mathrm{~h}$ após a adição da solução de hidróxido de amônio. Ao finalizar esta etapa o material foi decantado, retirou-se o sobrenadante e adicionouse um volume de água destilada igual ao volume da síntese. Este procedimento foi realizado em duplicata. $\mathrm{O}$ material foi seco a $100^{\circ} \mathrm{C}$ em estufa a vácuo. Após $8 \mathrm{~h}$ de secagem na estufa o material foi pulverizado com graal e pistilo de porcelana. Os materiais híbridos de Óxido de Ferro/PAni(ADBS) foram preparados com as seguintes proporções de ADBS:anilina, 1:1,5 e a concentração inicial de anilina foi de $0,05 \mathrm{~mol} \cdot \mathrm{L}^{-1}$.

O óxido de ferro $(5,00 \mathrm{~g})$ foi adicionado a um balão de fundo redondo de 3 bocas e capacidade de $2 \mathrm{~L}$, contendo o respectivo volume de água. A suspensão foi mantida sob agitação mecânica, $200 \mathrm{rpm}$, e resfriada a $4-6^{\circ} \mathrm{C}$ com o auxílio de banho de gelo com sal grosso. Em seguida adicionou-se o ADBS, e 10 minutos depois, a anilina. Como agente oxidante utilizou o persulfato de amônio na proporção 1:1,25 (anilina:PSA). O persulfato de amônio foi dissolvido em $50 \mathrm{~mL}$ de água destilada, sendo a solução adicionada gota a gota durante $30 \mathrm{~min}$, ao sistema reacional, para minimizar o efeito do aquecimento ${ }^{25}$. A agitação foi mantida por 4 horas. Após a agitação adicionou-se à suspensão formada, um volume de etanol comercial suficiente para formar uma solução $40 \%$ de etanol em água. A suspensão foi filtrada sob pressão reduzida, lavada com $200 \mathrm{~mL}$ de solução de etanol 40 $\%$ em água e seca em estufa a vácuo a $100{ }^{\circ} \mathrm{C}$. Foram preparados amostras, com as respectivas proporções em massa de óxido de ferro e polianilina, 1:2 para o Híbrido 1; 1:1,5 para o Híbrido 2; 1:1 para o Híbrido 3; 1,5:1 para 
o Híbrido 4 e 2:1 para o Híbrido 5. Estes materiais foram caracterizados por difração de raios X, espectroscopia de IV, espectroscopia Mössbauer, microscopia eletrônica de transmissão (MET) e análise termogravimétrica.

\section{Resultados e Discussão}

A Figura 1 mostra o difratograma de raios $\mathrm{X}$ do Óxido de Ferro. Através da comparação dos picos obtidos com os valores tabelados no "Joint Commitee on Powder Diffraction Standards - Powder Difraction File (JCPDS PDF)" foi possível concluir que a amostra é uma mistura de óxidos de ferro. A goethita $(\alpha-\mathrm{FeOOH})$ evidenciada pelos 5 picos de difração em graus $2 \Theta=21,4^{\circ}, 26,6^{\circ}$, $33,4^{\circ}, 36,9^{\circ}$ e $53,6^{\circ}$ (JCPDS - PDF \# 810462), a presença de hematita $\left(\alpha-\mathrm{Fe}_{2} \mathrm{O}_{3}\right)$ é demonstrada nos 5 picos de difração em graus $2 \Theta=33,4^{\circ}, 35,6^{\circ}, 53,6^{\circ}, 61,7^{\circ}$ e $63,4^{\circ}$ (JCPDS - PDF \# 871163), a maguemita $\left(\gamma-\mathrm{Fe}_{2} \mathrm{O}_{3}\right)$ é inferida aos 7 picos de difração em graus $2 \Theta=30,6^{\circ}$, $35,9^{\circ}, 43,4^{\circ}, 47,6^{\circ}, 57,7^{\circ}, 63,3^{\circ}$ e $69,2^{\circ}$ (JCPDS - PDF \# 391346) e por magnetita $\left(\mathrm{Fe}_{3} \mathrm{O}_{4}\right)$ indicada pelos 7 picos de difração em graus $2 \Theta=18,1^{\circ}, 30,6^{\circ}, 36,9^{\circ}, 43,4^{\circ}$, $53,6^{\circ}, 57,7^{\circ}, 63,3^{\circ}$ (JCPDS - PDF \# 880866).

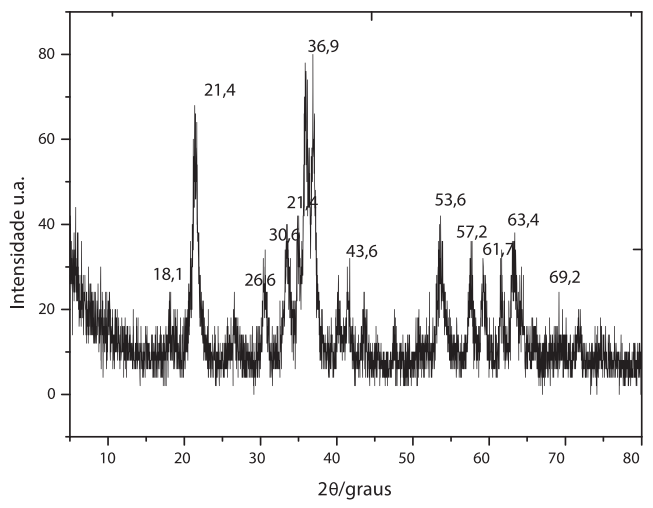

Figura 1. Difratogramas de raios X dos Óxidos de Ferro.

A Figura 2 mostra o espectro vibracional na região do infravermelho do Óxido de Ferro. As atribuições dos picos/bandas são mostrados na Tabela 1. A absorção em $1630 \mathrm{~cm}^{-1}$ evidencia água, a presença de goethita foi confirmada através das absorções em 893 e $798 \mathrm{~cm}^{-}$ ', atribuídos à deformação da ligação $\mathrm{Fe}-\mathrm{OH}$ no plano e fora do plano, respectivamente ${ }^{8}$. A absorção em $632 \mathrm{~cm}^{-1}$ é típica de estiramento $\mathrm{Fe}-\mathrm{O}$ no sítio tetraédrico da magnetita e maguemita que apresentam estrutura de espinélio ${ }^{21}$.
Tabela 1. Atribuição das bandas de absorção na região do infravermelho do Óxido de Ferro.

\begin{tabular}{|c|c|c|}
\hline $\mathbf{v} / \mathbf{c m}^{-1}$ & Amostras & Atribuições \\
\hline 1630 & $\mathrm{OF}^{5}$ & Estiramento O-H de água \\
\hline 893 & $\mathrm{OF}^{3}$ & $\begin{array}{c}\text { Estiramento Fe-OH no plano da } \\
\text { goethita }\end{array}$ \\
\hline 798 & $\mathrm{OF}^{3}$ & $\begin{array}{c}\text { Estiramento Fe-OH no fora do plano } \\
\text { da goethita }\end{array}$ \\
\hline 632 & $\mathrm{OF}^{21}$ & $\begin{array}{c}\text { Estiramento Fe-O do sítio tetraédrico } \\
\text { da magnetita e maguemita }\end{array}$ \\
\hline
\end{tabular}

A Figura 3 apresenta o espectro de Mössbauer da amostra de Óxido de Ferro obtido na temperatura ambiente (TA) ajustado com dois sextetos. A Figura 4 apresenta o espectro de Mössbauer da mesma amostra obtido na temperatura de nitrogênio líquido (NL) ajustado com três sextetos, utilizando o programa Mosswinn.

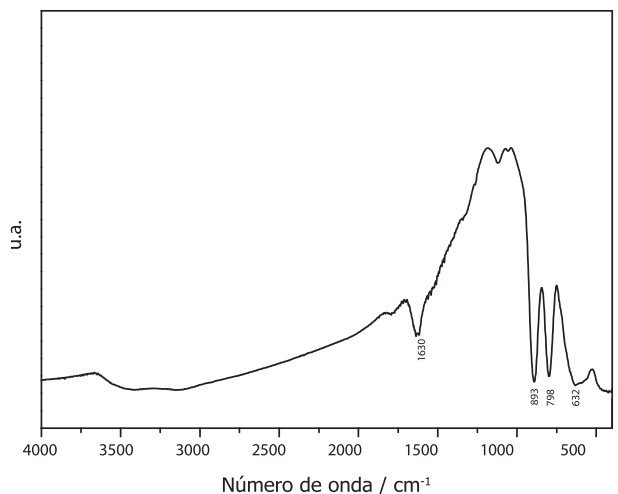

Figura 2. Espectro vibracional do na região do infravermelho do Óxido de Ferro.

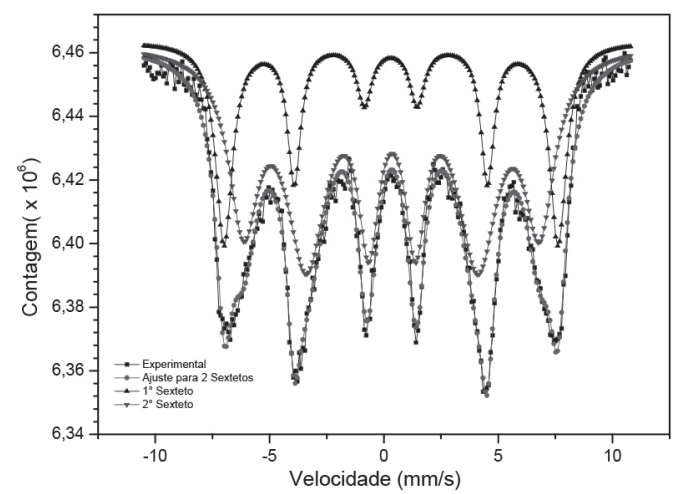

Figura 3. Espectro Mössbauer do Óxido de Ferro à temperatura ambiente. 


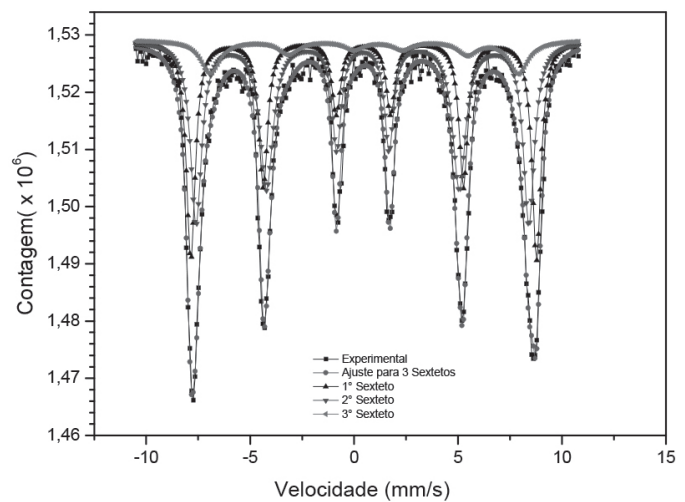

Figura 4. Espectro Mössbauer dos Óxidos de Ferro à temperatura de nitrogênio líquido.

A Tabela 2 apresenta os parâmetros hiperfinos obtidos do ajuste dos espectros Mössbauer para a amostra Óxido de Ferro. A análise do espectro Mössbauer a $300 \mathrm{~K}$ apresenta dois sextetos, podendo ser atribuído magnetita para o sexteto $1^{2}$ e goethita para $\mathrm{o}$ sexteto $2^{11}$. Já o espectro a $77 \mathrm{~K}$ apresenta três sextetos, sendo o sexteto 1 atribuído a maguemita ${ }^{22}$, o sexteto 2 a hematita ${ }^{27}$ e o sexteto 3 a goethita ${ }^{11}$.

Os valores obtidos nos deslocamentos isoméricos, nos desdobramentos quadrupolares e nos campos magnéticos são muito próximos aos valores apresentados na literatura para o sexteto 1 e 2 da análise a temperatura de nitrogênio líquido, indicando a presença de maguemita e hematita ${ }^{15}$. $\mathrm{O}$ sexteto 3 da amostra a temperatura de nitrogênio líquido e os sextetos da amostra à temperatura ambiente não foram encontrados valores idênticos, entretanto foi informado os valores mais próximos encontrados na literatura, correspondendo aos minerais magnetita e goethita. A presença de uma mistura de óxidos de ferro foi ratificada pelos resultados do difratograma de raios $\mathrm{X}$ e espectro de IV, conforme sugerido pela espectroscopia de Mössbauer.

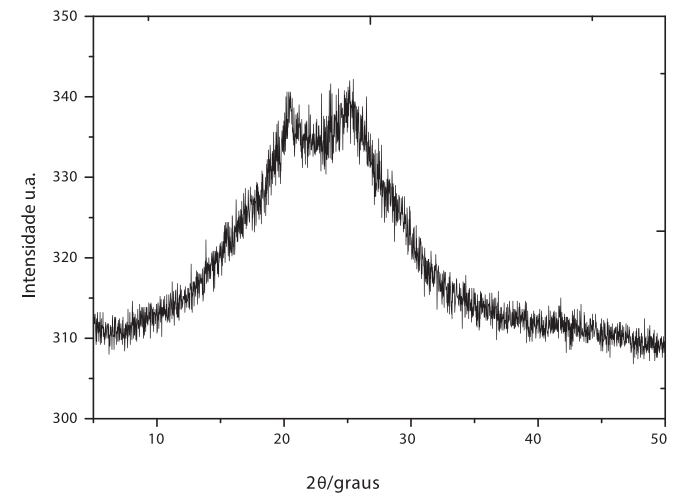

Figura 5. Difratograma de raios-X da PAni (ADBS).

A Figura 5 mostra o difratograma de raios $X$ da amostra PAni(ADBS). Observa-se dois picos centrados em $2 \Theta=20^{\circ}$ e $25^{\circ}$ atribuída às regiões cristalinas. Sobreposto aos picos ocorre um halo que é característico de materiais amorfos ${ }^{1}$.

O espectro vibracional na região de infravermelho da PAni (ADBS) é mostrado na Figura 6. Na Tabela 3 estão descritas as principais atribuições das vibrações

Tabela 2. Parâmetros Mössbauer da amostra de Óxidos de Ferro. TA = temperatura ambiente; NL = Temperatura á nitrogênio líquido; DI = deslocamento isomérico; $\mathrm{DQ}=$ desdobramento quadrupolar; $\mathrm{H}$ = campo magnético; A (\%) = área relativa.

\begin{tabular}{|c|c|c|c|c|c|c|}
\hline Amostra & Sítio & DI & DQ & H & Area & Mineral \\
\hline & & $(\mathrm{mm} / \mathrm{s})$ & $(\mathrm{mm} / \mathrm{s})$ & $(\mathrm{kOe})$ & $\%$ & \\
\hline Óxido de & Sexteto 1 & 0,31 & 0,021 & 454,2 & 26,9 & \\
\hline \multirow[t]{3}{*}{ Ferro (TA) } & Literatura $^{2}$ & $0,63 \pm 0,04$ & $0,08 \pm 0,04$ & $455 \pm 2$ & & Magnetita \\
\hline & Sexteto 2 & 0,35 & $-0,045$ & 402,5 & 73,1 & \\
\hline & Literatura $^{11}$ & $0,53 \pm 0,06$ & $0,00 \pm 0,1$ & $364 \pm 37$ & & Goethita \\
\hline Óxido de & Sexteto 1 & 0,46 & 0,013 & 516,9 & 39,4 & \\
\hline \multirow[t]{5}{*}{ Ferro (NL) } & Literatura $^{22}$ & $0,47 \pm 0,02$ & $0,08 \pm 0,02$ & $514 \pm 5$ & & Maguemita \\
\hline & Sexteto 2 & 0,41 & $-0,039$ & 497,6 & 50,0 & \\
\hline & Literatura $^{27}$ & $0,39 \pm 0,05$ & $-0,2 \pm 0,05$ & $497 \pm 5$ & & Hematita \\
\hline & Sexteto 3 & 0,82 & $-1,29$ & 461,0 & 10,6 & \\
\hline & Literatura $^{22}$ & $0,48 \pm 0,05$ & $-0,12 \pm 0,05$ & $460 \pm 4$ & & Goethita \\
\hline
\end{tabular}


da PAni (ADBS). A banda intensa na região de 4000 a $3600 \mathrm{~cm}^{-1}$ é atribuída a forma esmeraldina, normalmente esta banda sobrepõem aos picos entre 3500 a $3000 \mathrm{~cm}^{-1}$ atribuídos aos estiramentos de ligações N-H das aminas. Os Picos em 2962, 2927 e $2857 \mathrm{~cm}^{-1}$ típicos de ligações C-H de cadeias alifáticas foram atribuídas ao ADBS. Os picos em 1120, 1029, 1004, 740 e $700 \mathrm{~cm}^{-1}$ evidenciam a presença de ácidos sulfônicos e seus sais pelo grupo $\mathrm{SO}_{3}$.

Tabela 3. Atribuição das bandas de absorção na região do infravermelho da PAni(ADBS).

\begin{tabular}{|c|c|}
\hline $\mathrm{v} / \mathbf{c m}-1$ & Atribuições \\
\hline $\begin{array}{l}2962,2927 \\
\text { e } 2854\end{array}$ & Estiramento $\mathrm{C}-\mathrm{H}$ do ADBS \\
\hline 1560 & Estiramento $\mathrm{N}=\mathrm{Q}=\mathrm{N}$ e $\mathrm{C}=\mathrm{C}$ do anel quinóide $(\mathrm{Q})$ \\
\hline 1475 & $\begin{array}{l}\text { Estiramento } \mathrm{N}-\mathrm{B}-\mathrm{N} \text { e } \mathrm{C}=\mathrm{C} \text { do anel benzenóide } \\
\text { (B) }\end{array}$ \\
\hline 1292 & $\begin{array}{l}\text { Estiramento C-N (amina aromática) e deformação } \\
\text { C-C }\end{array}$ \\
\hline 1234 & Estiramento $\mathrm{C}-\mathrm{N}^{\circ+}$ (associado a condutividade) \\
\hline \multicolumn{2}{|l|}{1120} \\
\hline $\begin{array}{c}1029 \mathrm{e} \\
1004\end{array}$ & $\begin{array}{l}\text { Estiramento simétrico e assimétrico do } \mathrm{SO}_{3} \mathrm{e} \\
\text { estiramento S-Fenil (ADBS) }\end{array}$ \\
\hline 879 e 790 & $\begin{array}{l}\text { Deformação C-H fora do plano em anel } \\
\text { benzênico } p \text {-di-substituido }\end{array}$ \\
\hline 740 e 700 & $\begin{array}{l}\text { Deformação do grupo } \mathrm{SO}_{3} \text { em } \\
\text { alquilbenzenosulfonado }\end{array}$ \\
\hline 576 e 493 & Deformação fora do plano do anel aromático \\
\hline
\end{tabular}

A Figura 7 apresenta micrografias obtidas por microscopia eletrônica de transmissão (TEM) da PAni(ADBS), com resolução satisfatória para observação da morfologia do material formado, com aproximação de 10.000 vezes. Observa-se que os aglomerados de PAni(ADBS) ocorre na forma de bastões.

A Figura 8 mostra a curva termogravimétrica da amostra de PAni (ADBS), obtida sob atmosfera de oxigênio, com velocidade de aquecimento de 10 ${ }^{\circ} \mathrm{C} /$ min. A curva apresenta cinco etapas de perda de massa. A primeira etapa ocorreu até $180{ }^{\circ} \mathrm{C}$, sendo atribuído a pequenas moléculas do solvente, com 5,3 $\%$ de perda de massa. A segunda etapa ocorreu entre 180 e $335^{\circ} \mathrm{C}$ com perda de $11,4 \%$ de massa atribuído a volatilização ou degradação do ácido (ADBS) livre na molécula polimérica. A terceira faixa entre $335^{\circ} \mathrm{C}$ a $385^{\circ} \mathrm{C}$ com perda de $17,0 \%$ referente ao ácido na forma ligada. Posteriormente observou-se a quarta faixa de decomposição térmica entre 385 e $500^{\circ} \mathrm{C}$ com perda de aproximadamente $54,2 \%$, indicando a degradação da cadeia polimérica principal, e finalizando com a decomposição das cinzas até $630^{\circ} \mathrm{C} \operatorname{com} 12,1 \%$. A perda de massa foi de $100 \%$, devido à atmosfera de oxigênio, que promoveu uma oxidação completa da amostra.

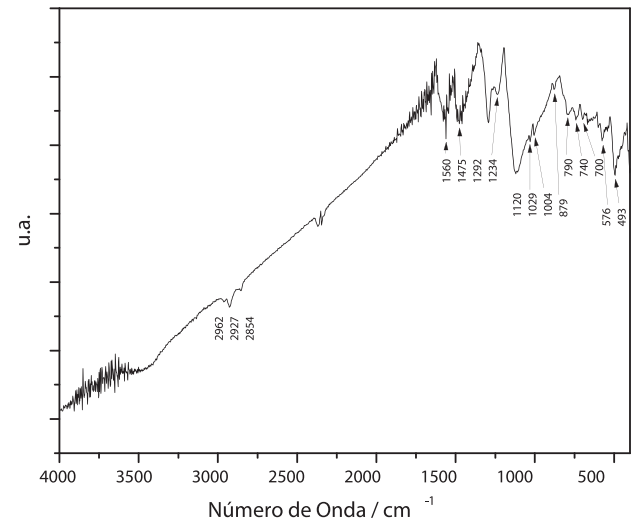

Figura 6. Espectro vibracional na região do infravermelho da PAni (ADBS).
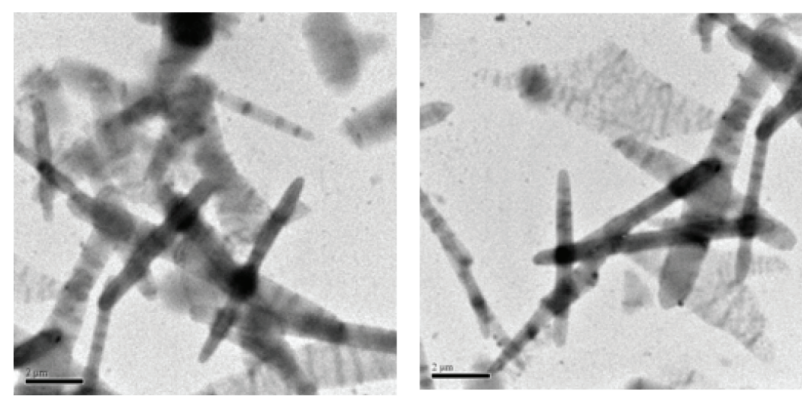

Figura 7. Micrografias obtidas por TEM da amostra de PAni(ADBS).

A Tabela 4 mostra os resultados de rendimento e condutividade elétrica. Rendimentos acima de 100 $\%$ são devidos ao excesso de ADBS na amostra. Observa-se também que ao ser adicionado o material inorgânico o rendimento da PAni aumenta, isto pode ser atribuído a uma possível ação catalítica do Óxido de Ferro. Observa-se que a presença de óxidos de ferro não reduz os valores de condutividade elétrica quando comparados à PAni(ADBS) pura. Os valores obtidos para a condutividade elétrica são condizentes com os valores encontrado na literatura ${ }^{1}$. 
Tabela 4. Valores de condutividade elétrica e rendimento dos materiais Híbridos e PAni(ADBS).

\begin{tabular}{|c|c|c|c|}
\hline Sínteses & OF:PAni & $\boldsymbol{\sigma} /$ S.cm- $^{\mathbf{1}}$ & $\mathbf{R} / \mathbf{\%}$ \\
\hline PAni(ADBS) & $0: 1$ & $5,1 \times 10^{-2} \pm 4,1 \times 10^{-3}$ & 76 \\
\hline Híbrido 1 & $1: 2$ & $6,2 \times 10^{-1} \pm 8,5 \times 10^{-2}$ & 93 \\
\hline Híbrido 2 & $1: 1,5$ & $7,4 \times 10^{-2} \pm 4,7 \times 10^{-3}$ & 93 \\
\hline Híbrido 4 & $1,5: 1$ & $7,3 \times 10^{-2} \pm 4,6 \times 10^{-3}$ & 107 \\
\hline Híbrido 5 & $2: 1$ & $1,9 \times 10^{-1} \pm 1,1 \times 10^{-2}$ & 101 \\
\hline
\end{tabular}

A Figura 9 mostra os difratogramas de raios $X$ das amostras sintetizadas. Foi possível identificar a presença de magnetita, de maguemita, de goethita e de hematita. A PAni(ADBS) é evidenciada no híbrido 1, com proporção de 1:2 de óxido de ferro por polianilina, através do halo amorfo em $2 \theta=20^{\circ}$ e no pico em $2 \theta=25^{\circ}$.

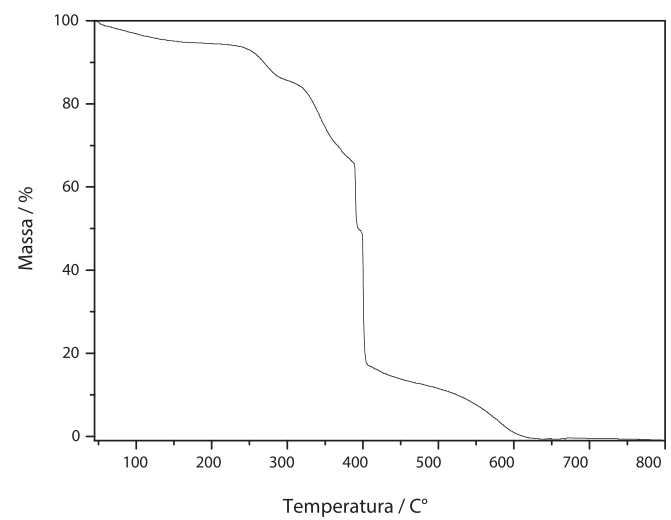

Figura 8. Curva termogravimétrica da amostra de PAni(ADBS).

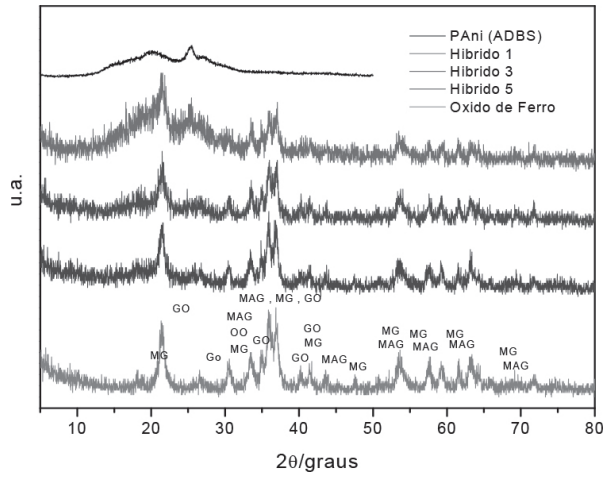

Figura 9. Difratogramas de raios X da PAni(ADBS), Híbridos e Óxido de Ferro.

A Figura 10 mostra o espectro de infra-vermelho do Híbrido 1, com atribuições elencadas na Tabela 5. Os picos 3414 e $3232 \mathrm{~cm}^{-1}$ são característicos de vibrações de estiramento simétrico e assimétrico do N-H associados a $\mathrm{NH}_{2}^{+}$. O ADBS é evidenciado no pico em $2926 \mathrm{~cm}^{-1}$ que corresponde ao estiramento $\mathrm{C}-\mathrm{H}$ de cadeias alifáticas. O Pico em $1630 \mathrm{~cm}^{-1}$ é característico de água ${ }^{5}$. Os picos em 1616, 1560 , e $1458 \mathrm{~cm}^{-1}$ evidenciam a presença de anéis quinóides e benzenóides da PAni. Na região de $1300 \mathrm{a} 1200 \mathrm{~cm}^{-1}$ indica a presenças de deformações tipo C-C e estiramentos C-N da amina aromática e associada a condutividade. Outra evidência de presença de ADBS são os estiramentos simétricos e assimétricos característicos do pico em $1124 \mathrm{~cm}^{-1}$. Os picos em 893 e $798 \mathrm{~cm}^{-1}$ estão associados a estiramento $\mathrm{Fe}-\mathrm{OH}$ da goethita e sobreposição da deformação $\mathrm{C}-\mathrm{H}$ fora do plano em anel benzênico $p$-di-substituido, no plano e fora do plano respectivamente. A magnetita e maguemita são identificadas pela absorção em $625 \mathrm{~cm}^{-1}$, característico do estiramento Fe-O do sítio tetraédrico da deformação fora do plano do anel aromático da PAni ${ }^{1,3}$.

Tabela 5. Atribuição das bandas de absorção na região do infravermelho do Híbrido 1.

\begin{tabular}{|c|c|}
\hline $\mathrm{v} / \mathbf{c m}^{-1}$ & Atribuições \\
\hline $\begin{array}{c}3414 \text { e } \\
3232\end{array}$ & $\begin{array}{l}\text { Vibrações de estiramento simétrico e assimétrico de } \\
\text { N-H }\end{array}$ \\
\hline 2926 & Estiramento C-H do ADBS \\
\hline 1637 & Estiramento O-H de água \\
\hline $\begin{array}{c}1616 \mathrm{e} \\
1560\end{array}$ & Estiramento $\mathrm{N}=\mathrm{Q}=\mathrm{N}$ e $\mathrm{C}=\mathrm{C}$ do anel quinóide $(\mathrm{Q})$ \\
\hline 1458 & Estiramento N-B-N e $\mathrm{C}=\mathrm{C}$ do anel benzenóide (B) \\
\hline 1296 & $\begin{array}{l}\text { Estiramento C-N (amina aromática) e deformação } \\
\text { C-C }\end{array}$ \\
\hline 1234 & Estiramento C-N ${ }^{\circ}$ (associado a condutividade) \\
\hline 1124 & $\begin{array}{l}\text { Estiramento simétrico e assimétrico do SO3 e } \\
\text { estiramento S-Fenil (ADBS) }\end{array}$ \\
\hline 893 & $\begin{array}{l}\text { Estiramento Fe-OH no plano da goethita e } \\
\text { sobreposição da deformação C-H fora do plano em } \\
\text { anel benzênico p-di-substituido }\end{array}$ \\
\hline 798 & $\begin{array}{l}\text { Estiramento Fe-OH no fora do plano da goethita e } \\
\text { sobreposição da deformação C-H fora do plano em } \\
\text { anel benzênico } p \text {-di-substituido }\end{array}$ \\
\hline 625 & $\begin{array}{l}\text { Estiramento Fe-O do sítio tetraédrico da magnetita } \\
\text { e maguemita e deformação fora do plano do anel } \\
\text { aromático da PAni }\end{array}$ \\
\hline
\end{tabular}

Nas Figuras 11 a 20 são mostrados os espectros de Mössbauer dos Híbridos 1, 2, 3, 4 e 5 obtidos à temperatura ambiente e à temperatura de nitrogênio líquido. 


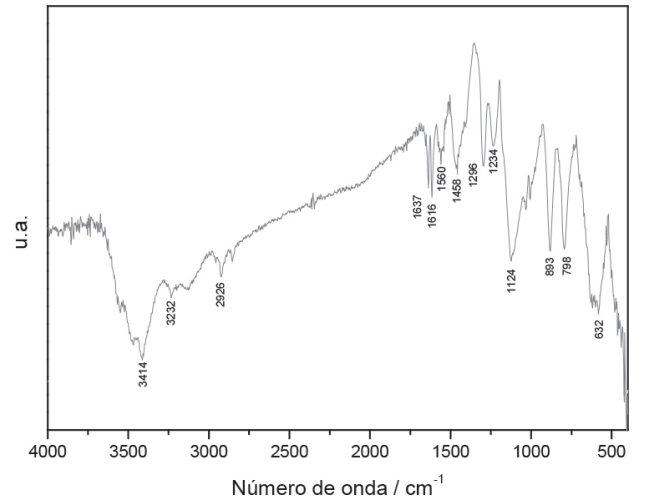

Figura 10. Espectro vibracional na região do infravermelho do Híbrido 1

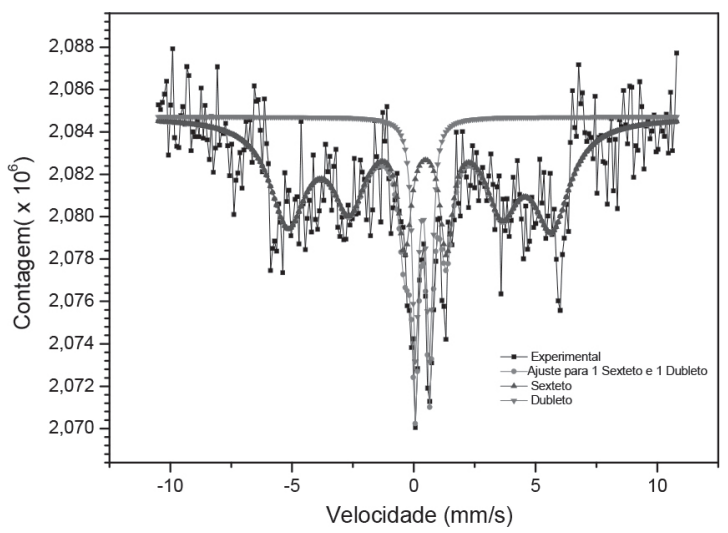

Figura 11. Espectro Mössbauer do Híbrido 1 à temperatura ambiente.

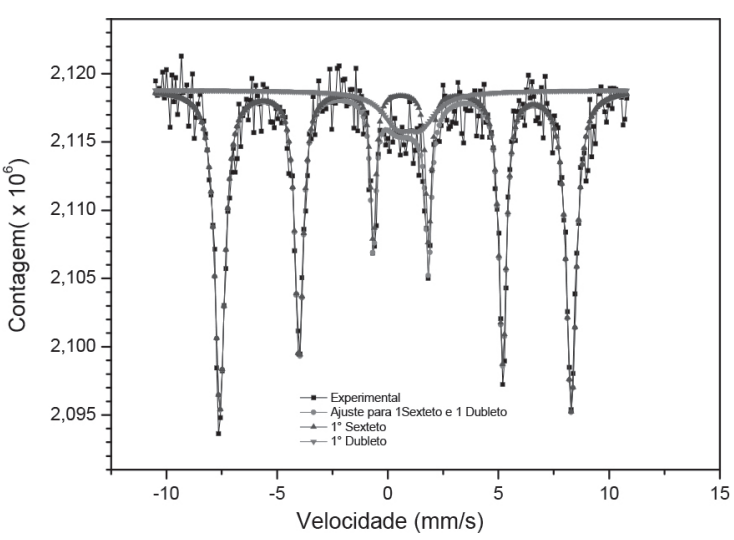

Figura 12. Espectro Mössbauer do Híbrido 1 à temperatura de nitrogênio líquido.

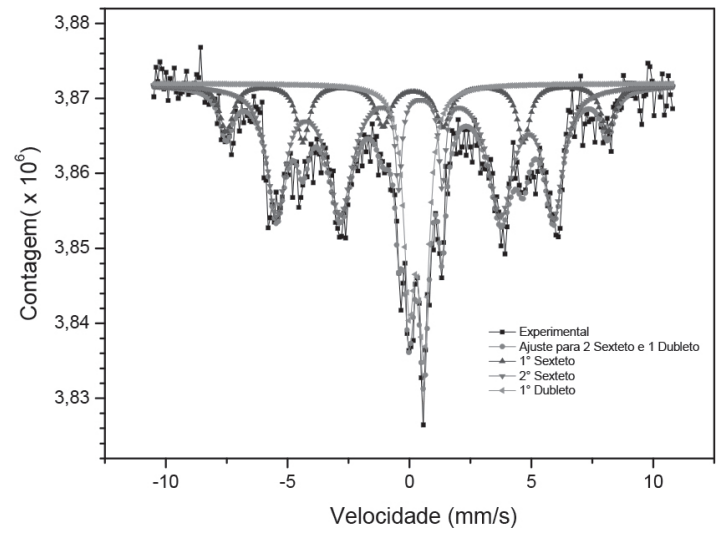

Figura 13. Espectro Mössbauer do Híbrido 2 à temperatura ambiente.

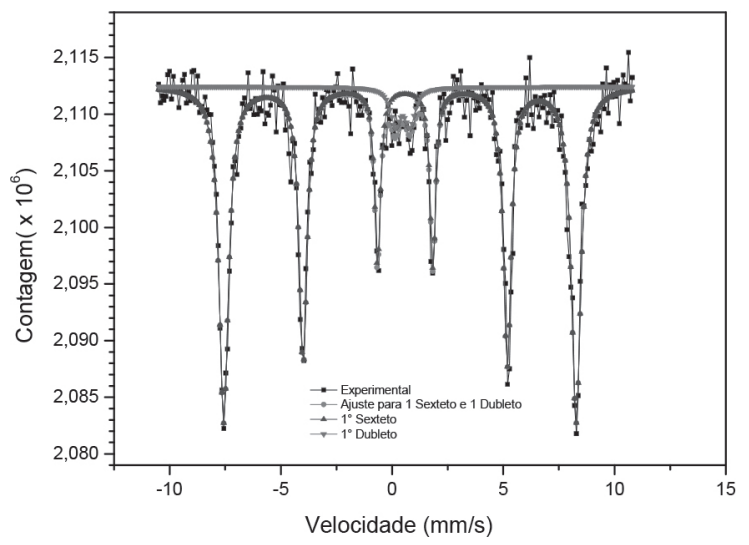

Figura 14. Espectro Mössbauer do Híbrido 2 à temperatura de nitrogênio líquido.

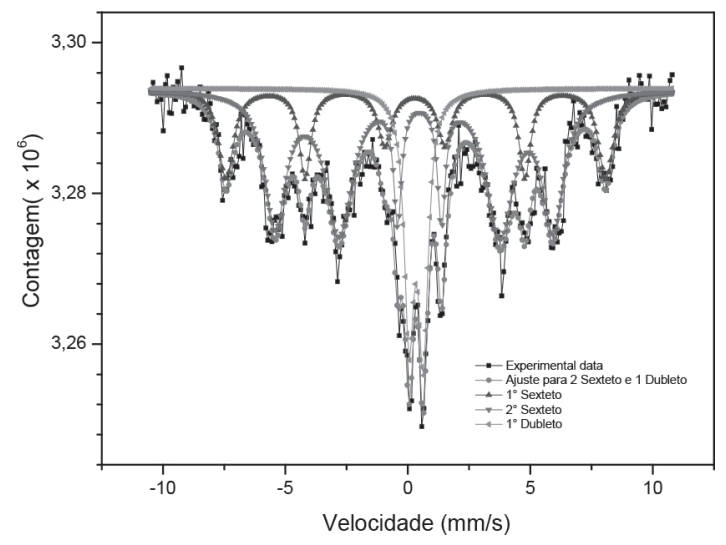

Figura 15. Espectro Mössbauer Híbrido 3 à temperatura ambiente. 


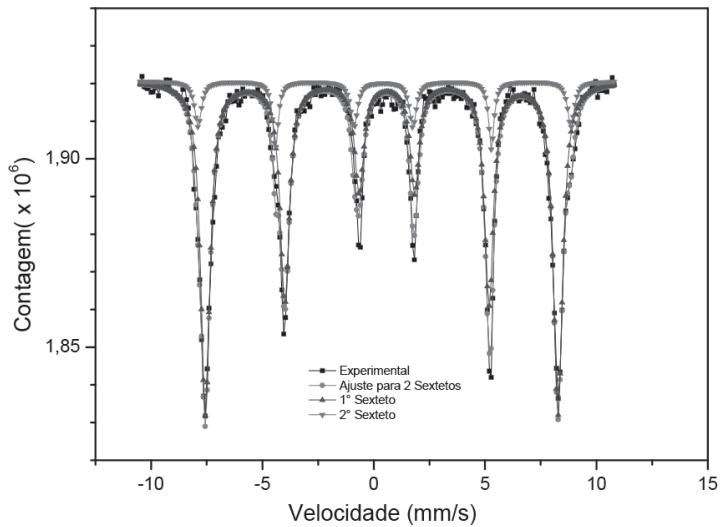

Figura 16. Espectro Mössbauer Híbrido 3 à temperatura de nitrogênio líquido.

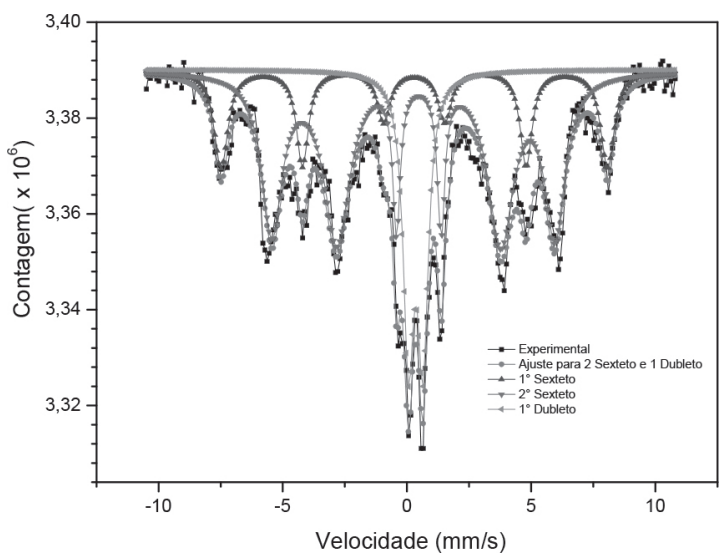

Figura 17. Espectro Mössbauer do Híbrido 4 à temperatura ambiente.

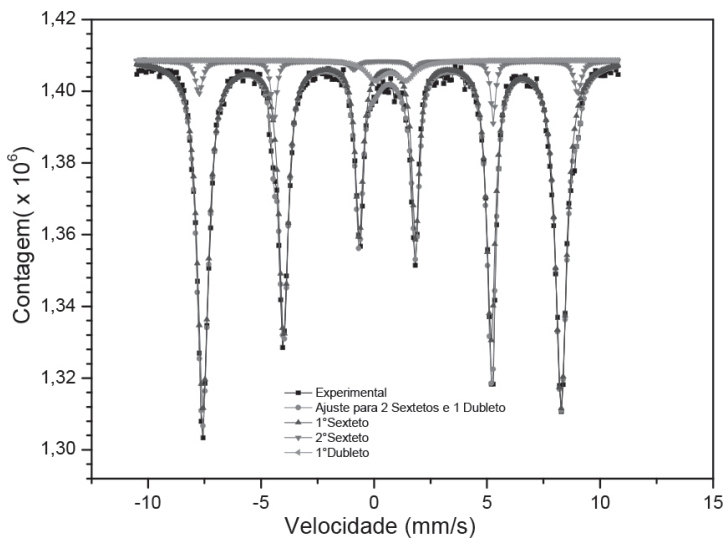

Figura 18. Espectro Mössbauer do Híbrido 4 à temperatura de nitrogênio líquido.

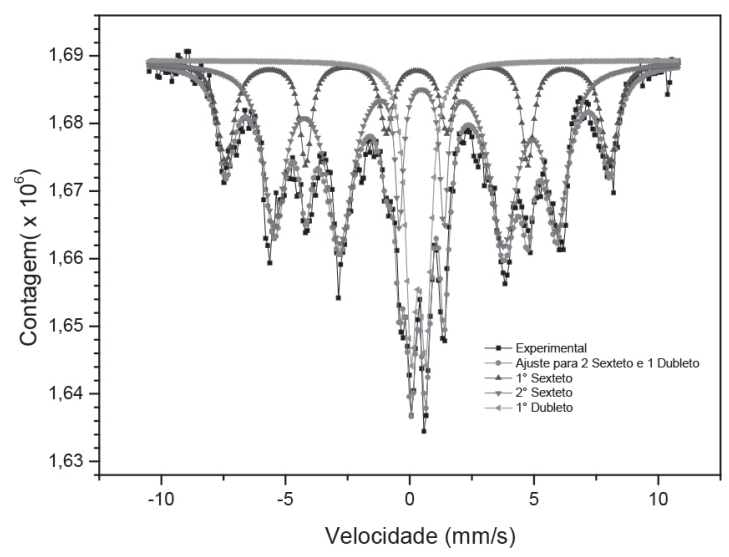

Figura 19. Espectro Mössbauer do Híbrido 5 à temperatura ambiente.

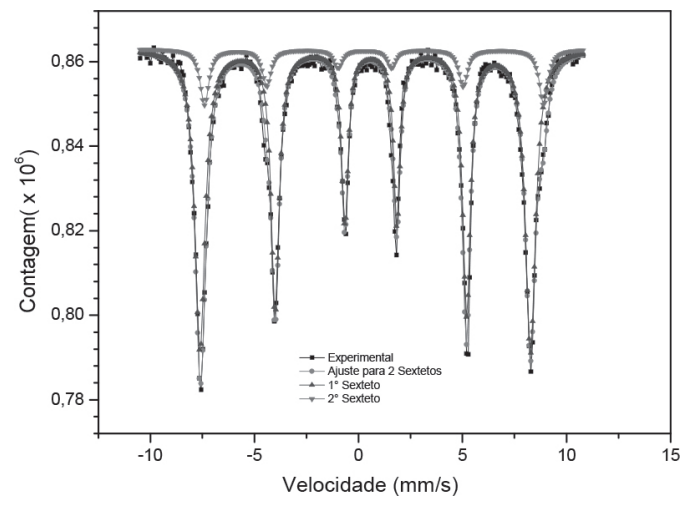

Figura 20. Espectro Mössbauer do Híbrido 5 à temperatura de nitrogênio líquido.

As Tabelas 6 e 7 mostram os parâmetros hiperfinos obtidos do ajuste dos espectros Mössbauer dos Híbridos 1, 2, 3, 4 e 5. O sexteto 2 do Híbrido 3 apresenta valores iguais aos apresentados na literatura indicando a presença de hematita ${ }^{9}$. Os dubletos evidenciam a presença de magnetita nas amostras, pois os valores são muito próximos aos informados na literatura ${ }^{10}$, valores muito próximos indicam que os átomos de $\mathrm{Fe}$ estão em ambientes químicos e magnéticos semelhantes ${ }^{15}$. Todas as amostras apresentaram dubletos em espectros obtidos na temperatura ambiente. Esta característica evidencia que o material é superparamagnético, ou seja, composto por nanopartículas, que devido ao seu tamanho reduzido, apresenta apenas um domínio 
Tabela 6. Parâmetros Mössbauer das amostras de Híbridos 1 a 5 á temperatura ambiente (RT). DI = deslocamento isomérico; DQ = desdobramento quadrupolar; $\mathrm{H}$ = campo magnético; $\mathrm{A}(\%)$ = área relativa.

\begin{tabular}{|c|c|c|c|c|c|}
\hline Amostra & Sítio & $\begin{array}{c}\text { DI } \\
(\mathrm{mm} / \mathrm{s})\end{array}$ & $\begin{array}{c}\mathrm{DQ} \\
(\mathrm{mm} / \mathrm{s})\end{array}$ & $\begin{array}{c}\text { H } \\
\text { (kOe) }\end{array}$ & $\begin{array}{c}\text { Área } \\
\%\end{array}$ \\
\hline \multirow[t]{2}{*}{ Híbrido 1} & Sexteto 1 & 0,37 & $-0,48$ & 336,8 & 84,6 \\
\hline & Dubleto 1 & 0,36 & 0,59 & - & 15,4 \\
\hline \multirow[t]{3}{*}{ Híbrido 2} & Sexteto 1 & 0,23 & 0,31 & 486,2 & 19,3 \\
\hline & Sexteto 2 & 0,34 & $-0,44$ & 353,5 & 58,1 \\
\hline & Dubleto 1 & 0,29 & 0,61 & - & 22,5 \\
\hline \multirow[t]{3}{*}{ Híbrido 3} & Sexteto 1 & 0,31 & 0,08 & 482,1 & 24,6 \\
\hline & Sexteto 2 & 0,37 & $-0,44$ & 352,6 & 57,4 \\
\hline & Dubleto 1 & 0,34 & 0,58 & - & 18,0 \\
\hline \multirow[t]{3}{*}{ Híbrido 4} & Sexteto 1 & 0,30 & 0,003 & 483,7 & 21,5 \\
\hline & Sexteto 2 & 0,37 & $-0,44$ & 354,2 & 59 \\
\hline & Dubleto 1 & 0,36 & 0,59 & - & 19,4 \\
\hline \multirow[t]{3}{*}{ Híbrido 5} & Sexteto 1 & 0,30 & 0,08 & 480 & 24 \\
\hline & Sexteto 2 & 0,35 & $-0,48$ & 353,6 & 58,3 \\
\hline & Dubleto 1 & 0,35 & 0,6 & - & 17,7 \\
\hline
\end{tabular}

Tabela 7. Parâmetros Mössbauer das amostras de Híbridos 1 a 5 á temperatura de nitrogênio líquido $(\mathrm{LN})$. $\mathrm{DI}=$ deslocamento isomérico; $\mathrm{DQ}=$ desdobramento quadrupolar; $\mathrm{H}=$ campo magnético; $\mathrm{A}(\%)$ = área relativa.

\begin{tabular}{|c|c|c|c|c|c|}
\hline Amostra & Sítio & $\begin{array}{c}\text { DI } \\
(\mathbf{m m} / \mathbf{s})\end{array}$ & $\begin{array}{c}\text { DQ } \\
(\mathbf{m m} / \mathbf{s})\end{array}$ & $\begin{array}{c}\text { H } \\
(\mathbf{k O e})\end{array}$ & $\begin{array}{c}\text { Área } \\
\mathbf{\%}\end{array}$ \\
\hline Híbrido 1 (LN) & Sexteto 1 & 0,47 & $-0,48$ & 493,6 & 87,4 \\
\hline & Dubleto 1 & 0,97 & 1,09 & - & 12,6 \\
\hline Híbrido 2 (LN) & Sexteto 1 & 0,47 & $-0,47$ & 492,5 & 94,6 \\
\hline & Dubleto 1 & 0,48 & 0,66 & - & 5,4 \\
\hline Híbrido 3 (LN) & Sexteto 1 & 0,47 & $-0,24$ & 492,4 & 85,4 \\
\hline & Sexteto 2 & 0,46 & 0,095 & 520,6 & 14,2 \\
\hline Híbrido 4 (LN) & Sexteto 1 & 0,47 & $-0,047$ & 492,6 & 88,8 \\
\hline & Sexteto 2 & 0,53 & 0,42 & 520,8 & 7,3 \\
\hline & Dubleto 1 & 0,72 & 1,36 & - & 3,9 \\
\hline Híbrido 5 (LN) & Sexteto 1 & 0,46 & $-0,55$ & 493,8 & 86,7 \\
\hline & Sexteto 2 & 0,53 & 0,44 & 506,7 & 13,3 \\
\hline
\end{tabular}

magnético ${ }^{12}$. Os Híbridos 2, 3, 4 e 5 apresentam valores próximos aos informados na literatura, mas não foram encontrados valores iguais para confirmação dos componentes da mistura.

Os dados do difratograma de raios $\mathrm{X}$ e espectro de infravermelho, avaliados anteriormente, evidenciam a presença de goethita, hematita, maguemita e magnetita, na amostra de Óxidos de Ferro. Estes minerais são os compostos que apresentam parâmetros mais próximos dos dados obtidos pela espectroscopia de Mössbauer, ratificando a informação do difratograma de raios-X e espectro de infravermelho. 


\section{Conclusão}

A PAni e seus híbridos foram sintetizados por via química, através da síntese do material inorgânico e posterior polimerização da anilina. As amostras de Óxido de Ferro/PAni(ADBS) não apresentaram alterações significativas de condutividade elétrica, demonstrando que a produção do híbrido não promove a perda da propriedade elétrica da PAni. O rendimento da reação de polimerização aumentou ao se adicionar o material inorgânico, devido a sua atuação como catalisador na reação.

\section{Referências}

1. Araújo, O. A.; Paoli, M.; Pilot plant scale preparation of dodecylbenzene sulfonic acid doped polyaniline in ethanol/water solution: Control of doping, reduction of purification time and of residues, Synthetic Metals, 2009.

2. Bahgat, A. A.; Fayek, M. K.; Salah, S.H.; Eissa, N. A.; Effect of the Divalent Iron on the Mossbauer Parameters of $\mathrm{Cox}(2+) \mathrm{Fe}(1-\mathrm{x})(2+)$ Fe2(3+)O4 Ferrites, Atomkernenergie 31, 276-9, 1978.

3. Bao, L.; Jiang, J. S.; Evolution of Microstructure and Phase of $\mathrm{Fe}_{3} \mathrm{O}_{4}$ in system of $\mathrm{Fe}_{3} \mathrm{O}_{4}$-polyaniline During High-energy Ball Milling, Physical B 367,182-187, 2005.

4. Bi, S.; Wei, X.; LI, N.; Lei, Z.; In-situ formation of $\mathrm{Fe}_{3} \mathrm{O}_{4}$ nanoparticles within the thermosensitive hairy hybrid particles, Materials Letters 62, 2963-2966, 2008.

5. Choi, J.; Lee, J. I.; Hong, J. H.; Kim, I. S.; Park, I. K.; Hur, N. H.; Immobilization of biomolecules on biotinylated magnetic ferrite nanoparticles, Chemical Physics Letters 428, 125-129, 2006.

6. Dias, J. C.; Martin, I. M.; Nohara, E. L.; Rezende. M. C.; Refletividade de Fótons Microondas por Tintas Poliuretâmicas Aditadas com Ferritas de NiZn e MnZn, Revista de Física Aplicada e Instrumentação, vol.18, no.I, 2005.

7. Dresco, P. A.; Zaitsev, V. S.; Gambino, R. J.; Chu, B.; Preparation and Properties of Magnetite and Polymer Magnetite Nanoparticles, Langmuir, 15, 1945-1951, 1999.

8. Gilbert, F.; Refait, P.; Lévêque, F.; Remazeilles, C.; Conforto, E.; Synthesis of goethite from $\mathrm{Fe}(\mathrm{OH})_{2}$ precipitates: Influence of $\mathrm{Fe}(\mathrm{II})$ concentration and stirring speed, Journals of Physics and Chemistry of Solids 69, 2124- 2130, 2008.

9. Gessa, C.; Melis, P.; Solinas, V.; Micera, G.; Burriesci, N.; Petrera, M.; Pflanzeneraehr, Z.; Iron Distribution in the Clay Fraction of Cambisols as Determined by Mossbauer Spectroscopy, X-ray Diffractometry and Selective Dissolution, Bodenk 147, 218-31, 1984.

10. Golden, D. C.; Ming, D. W.; Bowen, L. H.; Morris, R.V.; Lauer, H.V.; Acidified Oxalate and Dithionite Solubility and Color of
Synthetic, Partially Oxidized Al-Magnetites and Their Thermal Oxidation Products, Clays and Clay Miner. 42(1),53-62, 1994.

11. Go odman, B. A.; Lewis, G. D.; Mössbauer Spectra of Aluminous Goethites (alpha-FeOOH), J. Soil Sci. 32, 351-63, 1981.

12. Gonser, U.; Em Mössbauer Spectroscopy. Fugita, F. E.; Gonser, U.: Grant, R. W.; Gutlich, P.; Hafner, S. S.; Johnson, C. E., eds.; Springer-Verlag: Berlin, Heidelberg, New York, 1975.

13. Hosoro, T.; Takahashi, H.; Fugita, A.; Joseyphus, R. J.; Tohji, K.; Jeyadevan, B.; Synthesis of magnetite nanoparticles for AC magnetic heating, Journal of Magnetism and Magnetic Materials 321, 3019-3023, 2009.

14. Jiang, J.; AI, L.; LI, L.; Synthesis and magnetic performance of polyaniline/Mn- $\mathrm{Zn}$ ferrite nanocomposites with intrinsic conductivity, Springer Science-Business Media, 2009.

15. Leite, E. S.; Oliveira, A. C.; Garg, V. K.; Sartoratto, P. P. C.; Viali, W. R.; Morais, P. C.; Mössbauer characterization surface-coated magnetic nanoparticles for applications in transformers, Springer Science, 2009.

16. Li, L.; Jiang, J.; Xu, F.; Synthesis and ferrimagnetic properties of novel Sm-substituted LiNi ferrite-polyaniline nanocomposite, Materials Letters 61, 1091-1097, 2007.

17. Li, W. Qia O, X.; Zheng, Q.; Zhang, T.; One-step synthesis of $\mathrm{MFe}_{2} \mathrm{O}_{4}(\mathrm{M}=\mathrm{Fe}, \mathrm{Co})$ hollow spheres by template-free solvothermal method. Journal of Alloy and Compounds, v. 509, p. 6206-6211, 2011.

18. Long, Y.; Chen, Z.; Duvail, J. L.; Zhang, Z.; Wan, M.; Electrical and Magnetic Properties of Polyaniline/ $\mathrm{Fe}_{3} \mathrm{O}_{4}$ Nanostructures; Physica B 370, 121-130, 2005.

19. Lu, X.; Mao, H.; Chao, D.; Zhang, W.; Wei, Y.; Ultrasonic synthesis of polyaniline nanotubes containing $\mathrm{Fe}_{3} \mathrm{O}_{4}$ nanoparticles, Journal of Solid State Chemistry 179, 2609-2615, 2006.

20. Maity, D.; Choo, S. G.; Yi, J.; Ding, J. Xue, J. M.; Synthesis of magnetite nanoparticles via a solvent-free thermal, Journal of Magnetism and Magnetic Materials 321, 1256-1259, 2009.

21. Maity, D.; Agrawal, D. C.; Synthesis of iron oxide nanoparticles under oxidizing environment and their stabilization in aqueous and non-aqueous media, Journal of Magnetism and Magnetic Materials 308, 46-45, 2007.

22. Portnov, A. M.; Korovushkin, V. V.; Yakubovskaya, N. Y. Stable Maghemite in Weathered Crust of Yakutia. Dokl. Akad. Nauk 295, 196-9, 1987.

23. Rao, J. P.; Geckeler, K. E.; Polymer nanoparticles: Preparation techniques and size-control parameters. Progress in Polymer Science, v. 36, p. 887-913, 2011.

24. Silva, V. J.; Diniz, A. P .A.; Santos, P. T. A.; Vieira, D. A.; Costa, A. C. F. M.; Cornejo, D. R.; Gama, L.; Avaliação das características morfológicas e magnéticas de pós de ferrita $\mathrm{Ni}-\mathrm{Zn}$ dopadas com cromo, Revista Eletrônica de Materiais e Processos, v.1, 09-17, 2006. 


\section{Artigo Geral 5}

25. Stejskal, J.; Trchová, M.; Brodinová, J.; Kalenda, P.; Fedorava, S. V.; Prokes, J.; Zemek, j.; Coating of Zinc Ferrite Particles with a Conducting Polymer, Polyaniline, Journal of Colloid and Interface Science 298, 87-93, 2006.

26. Oh, J. K.; Park, J. M.; Iron oxide-based superparamagnetic polymeric nanomaterials: Design, preparation, and biomedical application. Progress In Polymer Science, v. 36, p. 168-189, 2011.

27. Vandenberghe, R. E.; Grave, E. D.; Geyter, G.D.; Landyat, C.; Characterization of goethite and hematite in a Tunisian soil profile by Mössbauer spectroscopy, Clays and Clay Miner 34, 275-80, 1986.

\section{Taciano P. Ferreira ${ }^{*}$ \& Olacir A. Araújo $^{2}$}

${ }^{1}$ Faculdade de Tecnologia SENAI Roberto Mange, CEP 75113-630, Anápolis, GO, Brasil.

${ }^{2}$ Ciências Exatas e Técnológicas, UEG, Caixa Postal 459, CEP 75001970, Anápolis-GO.

*e-mail: taciano80@hotmail.com 